\title{
Growing Burden of Stroke, Recent Advancements in Management and Global Commitments: The Way Forward
}

\author{
Vivek Nambiar ${ }^{1}$, Remya Sudevan², Amrutha Ajai ${ }^{3}$, Vijay Kumar Chattu, ${ }^{4, *}$ \\ ${ }^{l}$ Head of Division of Stroke, Centre of Neurosciences, Amrita Institute Medical Sciences, Ponekkara, Kochi, Kerala, INDIA. \\ ${ }^{2}$ Clinical Epidemiologist, Department of Health System Research, Amrita Institute of Medical Sciences, Ponekkara, Kochi, Kerala, \\ INDIA. \\ ${ }^{3}$ Research Fellow, Division of Stroke, Centre of Neurosciences, Amrita Institute of Medical Sciences, Ponekkara, Kochi, Kerala, \\ INDIA. \\ ${ }^{4}$ Lecturer-Public Health and Research Coordinator, Faculty of Medical Sciences, University of the West Indies, St. Augustine, \\ TRINIDAD AND TOBAGO.
}

Worldwide, cerebrovascular accidents (stroke) are the second leading cause of death and the third leading cause of disability. Strokes mainly affect individuals at the peak of their productive life. Despite its enormous impact on countries' socio-economic development, this growing crisis has received very little attention to date ${ }^{[1]}$ In order to achieve the UN Sustainable Development Goal 3 which aims for a 30\% reduction in non-communicable diseases by 2030 , it requires a substantial reduction in cardiovascular diseases. Especially the secondary prevention of recurrent myocardial infarction and stroke among those with known cardiovascular disease can reduce cardiovascular mortality and morbidity. The cost-effectiveness of secondary prevention of cardiovascular disease, ${ }^{[2]}$ coupled with lifestyle changes, has long been established and it is the need of the hour to apply the recent advancements in emergency management of stroke to decrease mortality, morbidity and improve the quality of life.

Stroke is a heterogeneous disorder encompassing cerebral infarction, intracerebral hemorrhage and subarachnoid hemorrhage. Stroke is the most common cause of preventable disability and one of the most common causes of mortality all over the world. ${ }^{[3]}$ Even though the age standardized mortality rate of stroke has shown a decline in the last two decades across the globe, the absolute incidence rate and proportion of disabled stroke patients as well as mortality due to stroke is increasing relentlessly. This can be attributed to the demographic and health transitions pertinent to the developing countries in specific..$^{[4]}$

As per the Global Burden of Diseases report 2013, the global stroke incidence was 10.3 million, mortality was 6.5 million, the stroke survivors were 25.7 million and DALYs were 113 million. The report emphasized the diverging trend in developed and developing nations in which a remarkable rise in DALYs and mortality were observed in developing countries and for developed countries the same had no quantifiable change. ${ }^{[5]}$ The economic burden of stroke management and post stroke care is enormous worldwide and accounts approximately 3-5\% of all health expenditure. This shows that the care of a stroke patient in the individual level, health system level and societal level is very expensive. ${ }^{[6]}$

Statistics shows an increasing incidence of stroke in developing countries like India where $22 \%$ of the population are below the poverty line. Cumulative incidence of stroke in India is estimated to be 105-152/100,000 persons per year during the last 20 years. ${ }^{[5,7]}$ The incidence of stroke in Indian scenario is at a much younger age compared to global occurrence. Due to the early occurrence, demographic transition and longer longevity of our population, the socioeconomic burden of this chronic condition is devastating. Despite the fact that 'time is brain' in acute stroke, the emergency management of acute stroke still lags behind myocardial infarction owing to multiple factors including the delay in bringing the patient to the hospital, logistic issues and fundamental issues in the health care system. ${ }^{[8,9]}$

With the emergence of newer imaging techniques like CT, MRI and PET, there is an immense progress in the diagnosis of stroke. In stroke management the key point to be addressed is the early evaluation and prevention of the development of brain ischemia. Brain ischemia leading to infarction is the contributor of all morbidity and even mortality of the affected individual. Among the three types of strokes, ischemic stroke accounts for $87 \%$ and the rest shared by transient ischemic attacks and hemorrhagic strokes. An ischemic stroke is often due to atherosclerosis and can be embolic also in $15 \%$ of cases because of atrial fibrillation. In ischemic hypoxic injury, molecular and metabolic events lead to cerebral injury in which there is a dense central core and a surrounding less dense zone of ischemia called penumbra. If the cerebral perfusion is not restored rapidly neuronal death occurs. Nevertheless, the penumbra zone nerve cells remain viable for 3- 4.5 $\mathrm{h}$ (therapeutic window). These neurons can be rescued by reperfusion and neuroprotective agents. ${ }^{[10]}$ Around $20-25 \%$ of strokes are wake up strokes and the exact time of onset of symptoms may be unknown, there by denying the affected patients from the benefits of intravenous thrombolysis as well as endovascular thrombectomy. ${ }^{[1]}$

Acute ischemic stroke management witnessed a paradigm shift with the initiation of thrombolysis in 1996 using intravenous tissue plasminogen activator (tPA). Evidences from trials prove that patients undergoing thrombolysis within the window period of $4.5 \mathrm{hrs}$ have a substantially better chance of functional independence with minimal or no disability after three months of treatment. However, no significant benefits in terms of functional outcome could be demonstrated in patients with large vessel occlusions after 3 months. Endovascular strategies yield higher rates of revascularisation as well as greater clinical outcomes in patients with large vessel occlusions when compared to intravenous thrombolysis alone. Evidences from studies have shown that rapid endovascular treatment improved the clinical outcomes as well as decreased the mortality rate in patients with acute ischemic stroke. Data from several trials have underlined the effectiveness of endovascular thrombectomy for internal carotid and proximal middle cerebral artery occlusions. ${ }^{[11]}$

A second landmark in the management of acute stroke in this scenario was the PROACT II trial, where intra-arterial pro urokinase given to large vessel occlusion leading to significant recanalization and outcome. Subsequently several endovascular modalities were invented including MERCI device/ Penumbra device with variable degree of recanalization but never had 


\section{Nambiar, et al.: Burden of Stroke and Recent Advancements}

significant clinical benefit compared to the intra venous thrombolysis. ${ }^{[12]}$ The incidental use of a solitaire stent for thrombectomy revolutionised endovascular treatment. In the stent retrieval method the deployed stent traps the clot which when removed opens up the artery and provides immediate revascularisation of occluded vessels ultimately leading to better clinical outcomes. Currently, Solitaire which was FDA approved in 2012 is the most commonly used stent retriever. Retrieval of the stent reduces the potential complications related with permanent stent implantation and associated ischemia and the use of long-term antiplatelet therapy. Also, they have a safety profile comparable to that of thrombolytic therapy. Though it is strongly recommended in all patients with a higher benefit to risk ratio the potential complications are intracranial haemorrhage, stent detachment, arterial dissection and puncture site thrombosis, infections etc. Reports have shown that the occurrences of these complications are rare in clinical practice. ${ }^{[12]}$ Financial affordability is another critical issue for repulsion from endovascular treatment. Compared to the cost of disability the cost for the procedure is reasonably accountable. In short, there should not be any hesitancy to proceed with endovascular thrombectomy in centres where sufficient infrastructure and expertise are available.

The crucial challenges in the implementation of acute stroke treatment methods like thrombolysis and mechanical thrombectomy are lack of comprehensive stroke treatment centres/stroke care centres in rural areas, improper and inefficient transport system and lack of experts in the primary level. The rural areas of developing nations like India are devoid of infrastructure, man power and logistic facilities. Majority of the functioning comprehensive stroke care centres are situated in the urban areas running under private sector. Travel time, distance and financial crisis of people with the disease make these centres inaccessible. Initiatives like Hub and spoke model can be made use in these areas by which all the peripheral centres are connected to a speciality centre through telemedicine or telestroke service and the expert advice could be obtained at any time. Despite the development in acute stroke care, stroke related disabilities are increasing and this will have a significant impact on family, healthcare system and economy. With a large proportion of patients with residual deficits post stroke, rehabilitation plays a key role in the recovery of the survivors. ${ }^{[13]}$ The WHO Global Monitoring Framework for Non-Communicable Diseases aims for at least $50 \%$ coverage of those eligible with drug therapy and counselling by 2025. To achieve this, the national governments must show a strong commitment for the health of its citizens. The countries within the regions should come together and think seriously to tackle the rising epidemic of strokes by strengthening of health systems. A successful global health diplomacy could bring in all the stakeholders (ministries, civil society organizations etc.) together as regional force with strong political commitment to address this critical issue and enact comprehensive policies as done at the Port of Spain declaration for the preventions of NCDs. ${ }^{[14]}$ Further, WHO should develop guidelines for the management of acute stroke in low- and middle-income countries, conduct capacity building programs in stroke prevention, treatment and rehabilitation through its partners for a greater impact.

\section{REFERENCES}

1. Johnson W, Onuma O, Owolabi M, Sachdev S. Stroke: a global response is needed. Bulletin of the World Health Organization. 2016;94(9):634.

2. Yusuf S, Islam S, Chow CK, Rangarajan S, Dagenais G, Diaz R, Gupta R, Kelishadi R, Iqbal R, Avezum A, Kruger A. Use of secondary prevention drugs for cardiovascular disease in the community in high-income, middle-income and low-income countries (the PURE Study): a prospective epidemiological survey. The Lancet. 2011;378(9798):1231-43.

3. Johnston SC, Mendis S, Mathers CD. Global variation in stroke burden and mortality: estimates from monitoring, surveillance and modelling. Lancet Neurol. 2009;8(4):345-54.

4. Adamson J, Beswick A, Ebrahim S. Is stroke the most common cause of disability?. J Stroke Cerebrovasc Dis. 2004;13(4):171-7.

5. Feigin VL, Krishnamurthi RV, Parmar P, Norrving B, Mensah GA, Bennett DA, et al. Update on the Global Burden of Ischemic and Hemorrhagic Stroke in 1990-2013: The GBD 2013 Study. Neuroepidemiology. 2015;45(3):161-76.

6. Rajsic S, Gothe H, Borba HH, Sroczynski G, Vujicic J, Toell T, et al. Economic burden of stroke: a systematic review on post-stroke care. Eur J Health Econ. 2018. doi: 10.1007/ s10198-018-0984-0

7. Pandian JD, Srikanth V, Read SJ, Thrift AG. Poverty and stroke in India: a time to act. Stroke. 2007;38(11):3063-9.

8. Anand K, Chowdhury D, Singh KB, Pandav CS, Kapoor SK. Estimation of mortality and morbidity due to strokes in India. Neuroepidemiology. 2001;20(3):208-11.

9. Srivastava AK, Prasad K. A study of factors delaying hospital arrival of patients with acute stroke. Neurol India. 2001;49(3):272-6.

10. Eugenio RR, Michael JR. New Developments in Stroke Rehabilitation. Current Atherosclerosis Reports. 2002;4(4):277-84.

11. National Institute of Neurological Disorders and Stroke rt-PA Stroke Study Group. Tissue plasminogen activator for acute ischemic stroke. New England Journal of Medicine. 1995;333(24):1581-8.

12. Goyal M, Demchuk AM, Menon BK, Eesa M, Rempel JL, Thornton J, et al. Randomized assessment of rapid endovascular treatment of ischemic stroke. New England Journal of Medicine. 2015;372(11):1019-30.

13. Brewer L, Horgan F, Hickey A, Williams D. Stroke rehabilitation: recent advances and future therapies. QJM: An International Journal of Medicine. 2012;106(1):11-25.

14. Chattu VK, Sakhamuri S. Port-of-Spain Declaration for global NCD prevention. The Lancet. 2018;391(10131):1682.

*Correspondence to:

Vijay Kumar Chattu,

Lecturer-Public Health and Research Coordinator, Faculty of Medical Sciences, University of the West Indies, St. Augustine, TRINIDAD AND TOBAGO.

Email:vijay.chattu@sta.uwi.edu

Cite this article as: Nambiar V, Sudevan R, Ajai A, Chattu VK. Growing Burden of Stroke, Recent Advancements in Management and Global Commitments: The Way Forward. J Pharm Pract Community Med. 2018;4(4):191-2. 